\title{
Study on Characteristics of the Wheelchair-User Combination
}

\author{
Zerui Xiang, Jinyi Zhi, Shiyu Dong, Bochu Xu \\ Institute of Design and Research for Man-Machine-Environment Engineering System, Southwest Jiaotong \\ University, Chengdu, China \\ Email: xiangzerui@163.com
}

Received 11 March 2016; accepted 19 May 2016; accepted 24 May 2016

\begin{abstract}
To summarize the characteristics of wheelchair-user combination, this paper studied wheelchairs, wheelchair users, nursing staffs and interaction relationship between them. Firstly, the findings of some research on wheelchairs and wheelchair users were investigated, and the studies in four aspects of wheelchairs and their accessories, wheelchair users, wheelchairs and wheelchair users (man-machine system), and skills training for the performance of wheelchair were reviewed. Then "wheelchair-user combination" was put forward based on the relationship between wheelchairs and wheelchair users in real life, and the "wheelchair-user combination" was divided into "WUs-Ws system" and "WUs-Ws-NSs system" according to whether the nursing staffs were needed. Finally, this paper analytically studied the characteristics of wheelchair users, characteristics in use of wheelchair and characteristics of nursing staffs, as well as put forward the characteristics of the wheelchair-user combination.
\end{abstract}

\section{Keywords}

Wheelchair-User Combination, Wheelchair Users, Nursing Staffs, Wheelchairs, Wheelchair Ergonomics

\section{Introduction}

The wheelchair refers to the chair with wheels movement, mainly including manual wheelchair, electric wheelchair and motor wheelchair. It can be used to improve movement for lower limb disabilities or others with difficulty in walk [1]. Wheelchair users (WUs) are mainly the persons using wheelchairs (Ws). In this paper, we analytically studied the characteristics of wheelchair functions, the composition and physical characteristics of the WUs from a systemic perspective, divided the WUs into populations with self-care ability and without selfcare ability, and then further constructed wheelchair-user combination (WUC): WUs-Ws-NSs (Nursing staffs) system. Finally, WUC and characteristics of all components were generally put forward by the studies on all components in the system.

\section{Literature Review}

It can be clearly found in related literatures that studies on WUs and Ws focus on four aspects, including studies 
on Ws and their accessories, WUs, Ws-WUC system (man-machine system) and training for the use skills of Ws.

\subsection{Studies on Ws and Their Accessories}

At present, the acknowledged earliest wheelchair was the chair with wheels in the world, which was found in the sculpture of sarcophagus in Northern-Southern Dynasties (around AD 525). In 1783, Dawson J. (British) invented a kind of three-wheel wheelchair called “Bath”. In 1881, the wheelchair, which installed manual propulsion parts on the rim, was born after a long period of improvement. In 1916, the wheelchair equipped with power came out in London. Afterwards, Jennings H. C. and Everest H. A. jointly invented a folding wheelchair with "X" structure. Klein G (Canadian) invented the first electric wheelchair, and the batch production of electric Ws started in 1956 [2]-[4]. In the 21st century, the smart (intelligent) wheelchair gradually became the research hotspot [5] [6]. Until now, there were two clearer research routes: one was that the Ws and their accessories were studied in a technology level [4] [7]-[15], and the other one was that the Ws were studied from human factors [16]-[20].

From the development process of Ws, each technological advance of wheelchair brings convenience to WUs from manual wheelchair, electric wheelchair, and motor wheelchair to smart wheelchair and improves the traffic capacity of WUs. These have important effect and significance in lowering the difficulties of moving barriers for WUs.

\subsection{Studies on WUs}

Perks et al. surveyed the WUs of Tayside in Scotland and finally obtained eighty effective interviews, and the interview content included user background detail, medical background and diagnosis, wheelchair and seating information, wheelchair environments and daily wheelchair activities [21]. Elaine et al. found that the physiological ability of WUs could be precisely quantified as well as pointed out that it was very important to precisely quantify the physiological ability of WUs by the test methods based on practical operation [22]. Moreover, Stanley et al. developed a kind of test tool called "wheelchair users functional assessment (WUFA)", and there were thirteen specific tasks to be tested in the tool [23]. Kilkens et al. further concluded that the test projects for the WUs mainly included wheelchair propulsion, transfer, curb, slope, track, sprint and wheelie [24]. They also proposed that the existing test projects should be integrated [25].

\subsection{Studies on WUs and Ws}

As early as in 1986, van der Woude analyzed the characteristics of both WUs and Ws from the constitution of man-machine systems, thought that Ws and WUs constituted the WUC, and then put forward "Wheelchair ergonomics (WE)” [26]. Afterwards, this theory was applied to the sports by Mason, thus "Conceptual model illustrating the factors that influence the ergonomics of sports wheelchair performance” was proposed [27]. Richter built a "Quasi-static wheelchair propulsion model” and defined the relationship among upper limbs, forelimbs (including hands), drive wheels and drive handles [28]. Likewise, in 1998, Rodgers et al. built a "Global and local coordinate systems for the wheelchair propulsion model”, developed a set of measurement system to catch posture via marked points, cameras, and so on, and then used this system to conduct three-dimensional dynamic analysis on the propulsion process of WUs [29]. Furthermore, Kotajarvi et al. used ten cameras motion capture system (Expert vision System) of Motion Analysis Corporation to collect the postures of wheelchair propulsion action [30]. Similarly, Finley et al. used the three-dimensional action analysis system (Motus video acquisition system) of PEAK Inc. to analyze the biomechanics of wheelchair propulsion action [31]. Subsequently, Yang used the motion analysis system (OPTOTRAK 3020) of Canada Northern Digital Inc. to capture and analyze the postures of wheelchair propulsion action [32].

In all the types of Ws, operational action of manual Ws in the propulsion process is the most complex and the hardest. Therefore, there have been some literatures which took WUs as an example to study the man-machine relationship between patients and Ws as well as the biomechanical characteristics in the propulsion process. During the process of their studies, the capture and analysis for postures of propulsion action were mainly accomplished by self-developed or commercial three-dimensional action analysis system. There has been manwheelchair relationship in the existing literatures, but there is still lack of a mode needing NSs to push WUs 
forward. If the nursing staff to push Ws should be considered, the whole wheelchair system may include patients, Ws, and NSs. So our paper will focus on studying and discussing the three components.

\subsection{Skills Training for Using Ws}

Wheelchair skills program (WSP) was a scheme for assessment and training as well as started in 1996, mainly including Wheelchair skills test (WST), Wheelchair skills test questionnaire (WST-Q), and Wheelchair skills training program (WSTP). At present, this was a more influential project in the world and has been widely accepted and adopted by developed countries in Europe, India, Bosnia, and so on. Moreover, this project not only focused on the WUs but also the nursing staffs [33] [34]. Comprehensive operational guidelines were formulated for WUs with a traumatic brain injury or spinal cord injury in literature [35], which was worth being referenced.

\section{Composition of Wheelchair-User Combination}

\subsection{Basic Definition of WUs}

Figure 1 shows the typical representatives of WUs, including the elderly with lower limb disability (the old women shown in Figure 1(a) and Figure 1(b)), the persons with injuries in lower limbs or trunks (the woman shown in Figure 1(c)), or the disabled (as shown in Figure 1(d), Figure 1(e) and Figure 1(f)), as well as the persons to provide special care or aid (the men shown in Figure 1(a) and Figure 1(c)). They all have direct interaction relationship with Ws and are the direct WUs. In order to effectively distinguish the two, we define the persons relying on the wheelchairs to arrive at their destinations as WUs and the other ones providing special care and aid as NSs. Furthermore, the WUs refer to the populations using Ws as the tools for accessibility in daily life, related activities and social affairs.

\subsection{Wheelchair-User Combination}

Ws, WUs and NSs constitute interactional "Wheelchair-user combination, WUC". Moreover, the new system is composed of WUs, Ws and NSs. In addition, the WUC includes not only the "WUs-Ws-NSs system" composed of WUs, Ws and NSs, but also the "WUs-Ws system" that some WUs have the stronger self-care abilities and do not need special nursing staffs. Figure 2 shows the composition of WUC and can reflect the basic relationship among WUs, Ws and NSs.

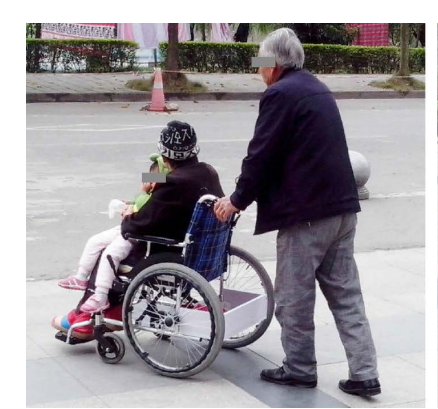

(a)

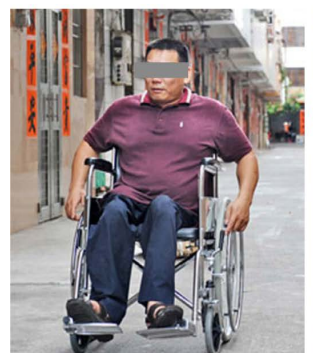

(d)

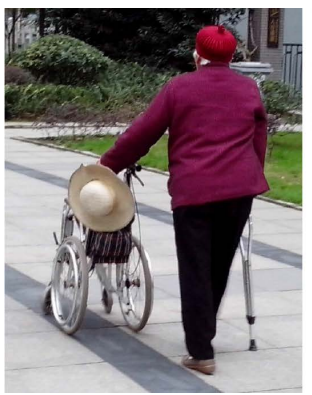

(b)

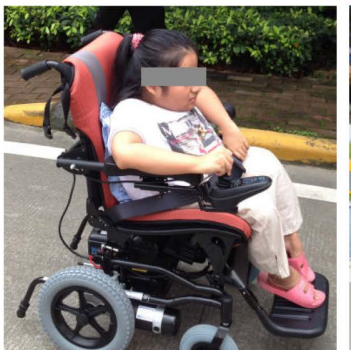

(e)

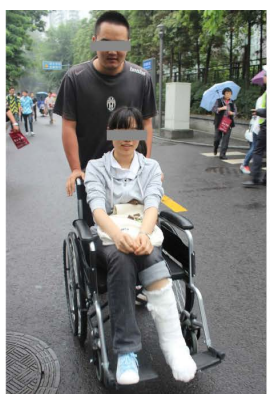

(c)

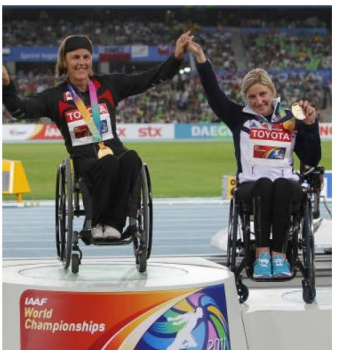

(f)

Figure 1. Some typical WUs. 


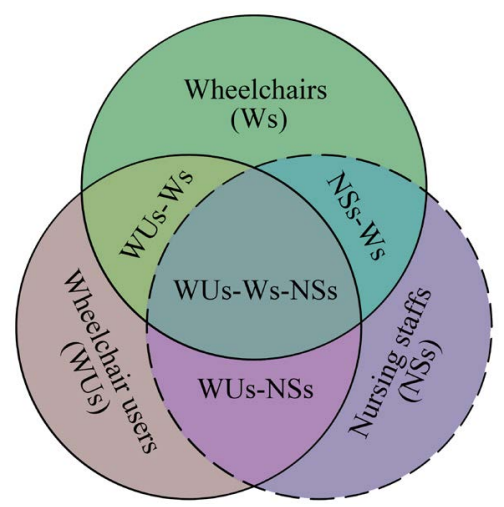

Figure 2. Composition schematic diagram of WUC.

In WUC, the WUs are the core element, and the Ws and NSs are the auxiliary elements. The WUs lose or partly lose ability to walk or related control ability due to the trouble of lower limbs, injuries in lower limbs or trunks, or disabilities, so they have to depend on Ws or NSs for the accessibility or usability. In essence, the Ws and NSs are the directly effective ways to improve comprehensive abilities of the WUs.

\section{Characteristic of WUC}

\subsection{Characteristic of WUs in WUC}

Any one of normal persons may face a possibility to be a WU due to natural aging, accident, illness, pregnancy, and so on. Some congenital or acquired troubles in the bodies of WUs result in the decline or loss of action activity and daily living activity, so they have to use the auxiliary mobile function of Ws to achieve the accessibility purpose of life, contacts, and participation in social affairs. Therefore, WUs have some common features as follows. The aging may result in a decline in physical strength. The whole body or part of the body may be paralyzed. Functions may be reduced or lost. The brain may lose the control ability for coordination or balance. The waist and lower limb may be congenitally deformed, injured, or disabled. In addition, activities of daily living (ADL) may be declined or lost. More specifically, there may be appeared one or more kinds of problems in each part of the human body in the process of information processing, as follows. The brain function to control body balance may be weakened or lost; visual function of eyes may be weakened or lost; support functions of the trunk (waist), thighs, calves or soles (of the feet) may be weakened or lost; and functions of hip joint, knee joint, or ankle joint may be weakened or lost.

The assessment of ADL has very close relationship with functions of limbs and the whole body movement. Twenty-one functional evaluation contents [36] in ADL are often used, and the main contents focusing on daily activity abilities include walk, transfer, wheelchair activities, change in position difference (position movement), activities on bed, verbal communication, activities of the hands, and writing. However, the main contents focusing on the daily living abilities include dressing, bathing, eating, going to lavatories (toilet), neatness and modification, controlling urination or defecation, household activities, using the phone (many contents belonged to the "self-care"). In addition, travel, psychological status, sensory introduction and others are involved in daily activity and daily living abilities. In real life, the different physical qualities and physical conditions of WUs may cause different movement abilities and daily living abilities. Thus the WUs can be divided into two groups according to whether they need the aid of NSs. Namely, one group refers to the WUs with the independent ADL abilities, and the other one refers to the WUs without the independent ADL abilities, thus needing aid of NSs.

\subsubsection{Characteristic of WUs with Independent ADL}

The WUs with independent ADL have the strongest comprehensive abilities in all the WUs. They usually have the abilities close to the normal people, so they have not only the normal information processing ability but also basic daily activity ability and daily living ability. Hence, the characteristics of WUs with independent ADL should be:

On the one hand, they should have more complete information processing abilities as follows. The physical strength should be relatively abundant enough to perform the instruction from the brain; the brain function to 
control body balance may be weakened, but this will not affect the information processing and decision-making; the receptors, such as eyes, ears, and so on, should have a certain function to obtain information; the upper limbs can move to a certain extent; the waist and lower limbs should be congenitally deformed, injured, or disabled and only affect movement. More concretely, the brain should be normal and physical strength should be abundant; upper limb functions should be relatively sound; the trunk may be abnormal, however, this will slightly affect the body balance and limbs coordination; thighs, calves, and the soles of feet may be disabled; and the functions of hip joint, knee joint, or ankle joint may be weakened or lost.

On the other hand, they should have more complete abilities in daily activities and daily living. Namely, they can take a short walk (including pushing Ws or walking with the aid of tools), transfer from Ws to other seats, use Ws for movement and position adjustment, turn over, and sit up from clinostatism. They have the necessary language communication ability, and the hands have activity ability and writing ability. As well as they can dress themselves, take a bath, take foods, go to the toilet, wash their faces, comb their hair, brush their teeth, shave, phone, and so on. Furthermore, they have relatively complete abilities to aware information, healthy psychology, and etc.

\subsubsection{Characteristic of WUs with the Aid of NSs}

WUs, who need the aid of NSs, are the populations with serious decrease or loss in one or more abilities of ADL. Compared with the WUs with independent abilities of ADL, there are some significant differences in information processing capability, daily activity ability and daily living ability. In addition to depending on Ws for movement, they have to rely on the NSs to provide the aid, or they cannot live by themselves. Hence, the characteristic of WUs with the aid of NSs should be:

One or more abilities may be damaged or lost in the process of information processing: the body may be frail and the physical strength may be seriously insufficient, the information processing and decision-making abilities of brain may be seriously damaged or missing; the information acquisition function of the receptors, such as eyes, ears and so on, may be weakened or lost; activity abilities of upper limbs may be weakened or lost; the waist and lower limbs may be congenitally deformed, injured, or disabled, thus affecting movement. More concretely, the brain may be abnormal; physical strength may be insufficient; upper limbs functions may seriously lose; the trunk may be abnormal, which has a major influence on body balance and limbs coordination; thighs, calves, and the soles of feet may be disabled; the functions of hip joint, knee joint, or ankle joint may be weaken or lose. Hence, it is necessary to use the Ws for movement and need NSs for daily activities and daily living.

Furthermore, one or more ability of daily activities and daily living may be damaged or lost. Namely, they cannot take a short walk (even pushing Ws or walking with the aid of tools), transfer from Ws to other seats, use Ws for movement and position adjustment, turn over, and sit up from clinostatism. They have no necessary language communication ability, and the hands have no activity ability or writing ability. Moreover, they cannot control urination or defecation, dress themselves, take a bath, take foods, go to the toilet, wash their faces, comb their hair, brush their teeth, shave, phone, and so on, as well as they have no relatively complete abilities to aware information, healthy psychology, and etc.

\subsection{Application Characteristics of Ws in WUC}

Whether the Ws are manual, electric, motor or smart (the smart wheelchair is still in the experimental research stage and has not been widely used, so this paper will not analyze and discuss its characteristics), they are used for the same purpose to relieve and decrease operational difficulty and service load as well as to be able to provide effective and convenient aid movement for the disabled populations with the decline or loss of moving ability. However, different kinds of Ws have different requirements for the abilities of Ws, and they have their own features:

1) Application characteristics of manual wheelchairs

Manual wheelchair has many advantages, for example, its structure is simple, its overall weight is lighter, the price is relatively cheap, and the maintenance and use are more convenient. However, only the WUs have a certain physical reserves, can the Ws be used? Moreover, the WUs have to depend on their hands or the power of NSs to drive and control the advance of Ws. Hence, this kind of Ws was suitable for all types of disabilities.

2) Application characteristics of electric wheelchairs

Compared with the manual wheelchair, the electric wheelchair can overcome the disadvantage needing hu- 
man power and provide electric energy reserves. Moreover, the WUs can use electric power to drive and control the Ws. However, because the installation of electric energy reserves system and relevant controllers, the electric wheelchairs are usually heavier and more expensive, and their maintenance is more complex. And above all, this kind of Ws has a higher requirement for the comprehensive information processing ability of the brain. The hands (at least one hand) can do flexible activities, or it is difficult to drive and control the electric wheelchair by controllers (or control leader).

3) Application characteristics of motor wheelchairs

Motor Ws have the engines and took the fuel as the power. They were divided into portable motor Ws and ordinary motor Ws [37], and they can overcome the manual Ws deficiency needing human power to drive. Compared with electric and manual Ws, the structure of motor Ws is more complicated, the overall weight is heavier, and the overall dimension is up to $2500 \mathrm{~mm} \times 1200 \mathrm{~mm} \times 1400 \mathrm{~mm}$. Furthermore, the speed can also be up to $50 \mathrm{~km} / \mathrm{h}$, and the use and maintenance are more difficult. The motor Ws can run through the roads like motor vehicles and need pay close attention to a variety of transportation signals and transportation routes in real time; hence, they had higher requirements for operation and driving by the WUs. Both the information processing ability of the brain and flexible operation functions of their hands should be approximately the same as those of ordinary people, otherwise it is difficult for them to safely drive and control the motor Ws.

4) Comparison for application characteristics of three kinds of Ws

To sum up, no matter what kinds of the Ws they are, the purpose is to provide a tool movement so as to accomplish the accessibility purpose. We comparatively analyzed three kinds of Ws from the perspective of use, and then the comparison order about the physical strength requirement, information processing and decision-making abilities of the brain, the flexible operation requirement of hands and the NSs demand, as well as the weight, overall dimensions, popularity degree of use, and the application scope of the Ws, and so on were shown in Table 1. It can be clearly found in Table 1 that the manual Ws need power of WUs, but their requirements in information processing and decision-making abilities of the brain and flexible operation of hands are lower. In addition, their weight is light, and the overall dimensions are small. They are used conveniently and flexibly and have been widely used, thus being used universally in real life. In comparison, the operation of manual wheelchair in the propulsion process is the most complex and most difficult, and the man-machine relationship between the Ws and WUs are more complex. In this paper, we define the tools similar to the Ws to provide aid for the disabilities as instrumental auxiliary ability provided by various STs.

\subsection{Characteristics of NSs in WUC}

Usually, the nursing staff has the sound physiology and psychology as well as abundant physical strength, so as to be able to provide the aid for WUs. NSs clearly understood the characteristics of WUs and knew the weakening and loss of their daily activity and daily living abilities, thus they can implement all kinds of the aid measures purposefully and timely. Simultaneously, some NSs have been specially trained in skills and nursing. In addition to providing the aid for the daily activities and daily living, they can correctly give the WUs first aid in the case of emergency (such as sudden illness). In general, the NSs would increase the ability of ADL of WUs and solve various barriers stemming from the weakening and loss of daily activity and daily living abilities of WUs via various aid measures. In this paper, we define the aid for the disabilities from NSs as service auxiliary ability provided by NSs.

Table 1. Comparison for application characteristics of three kinds of Ws.

\begin{tabular}{cccccc}
\hline Items & $\begin{array}{c}\text { Physical } \\
\text { strength } \\
\text { requirement }\end{array}$ & $\begin{array}{c}\text { Requirement of } \\
\text { information processing } \\
\text { and decision-making } \\
\text { abilities of the brain }\end{array}$ & $\begin{array}{c}\text { Requirement } \\
\text { of flexible } \\
\text { operation } \\
\text { of hands }\end{array}$ & $\begin{array}{c}\text { NSs } \\
\text { demand }\end{array}$ & $\begin{array}{c}\text { Weight } \\
\text { Overall } \\
\text { dimensions } \\
\text { Manual Ws }\end{array}$ \\
Electric Ws & 0 & 0 & 0 & $\bullet$ & 0 \\
degree \\
of use \\
Motor Ws
\end{tabular}

Note: “•” represented high, great, difficult, and severe degrees; “@” represented moderate degree; “○” represented low, small, easy, and light degrees. 


\subsection{Characteristics of WUC}

In WUC, the WUs are core element, and their own abilities determine the types of Ws as well as whether the NSs are needed. For the WUs with independent ability of ADL, they just need the controllable Ws to solve the barriers faced by themselves. However, for the WUs needing the special NSs, they have to need the aid of Ws and NSs to solve barriers faced by themselves due to the serious weakening or loss of one or more daily activity abilities and daily living abilities.

1) Characteristics of WUs-Ws system

The main characteristics of WUC with independent abilities of ADL (WUs-Ws system) are as follows. This system is composed of WUs and a certain type of Ws. The WUs can use the Ws to transfer from the Ws to other seats independently and use the Ws for movement and position adjustment. Moreover, they can turn over and sit up from clinostatism. They have the necessary language communication ability, and the hands have activity ability and writing ability. As well as they can control urination or defecation, dress themselves, take a bath, take foods, go to the toilet, wash their faces, comb their hair, brush their teeth, shave, phone, and so on. Furthermore, they have relatively complete abilities to aware information, healthy psychology, and etc. Hence, the comprehensive abilities of WUs in WUs-Ws system are the incorporation of their own abilities and instrumental auxiliary abilities from Ws.

2) Characteristics of WUs-Ws-NSs system

The main characteristics of WUC without independent abilities of ADL (WUs-Ws-NSs system) are as follows. This system is composed of WUs, NSs and a certain type of Ws. Without the aid of Ws and WUs, the WUs cannot solve the barriers faced by themselves. Hence, there may be one or more problems in this system, as follows. They cannot mutually transfer from the Ws to other seats and cannot use the Ws for movement and position adjustment. Moreover, they cannot turn over and sit up from clinostatism. They have no necessary language communication ability, and the hands have no activity ability or writing ability. As well as they cannot control urination or defecation, dress themselves, take a bath, take foods, go to the toilet, wash their faces, comb their hair, brush their teeth, shave, phone, and so on. Furthermore, they have no relatively complete abilities to aware information, healthy psychology, and etc. Therefore, the comprehensive abilities of WUs in WUs-Ws-NSs System are the incorporation of their own abilities, instrumental auxiliary abilities from Ws and the service auxiliary abilities from NSs.

\section{Conclusion}

According to the characteristics and composition of WUs in real life, this paper proposed that the WUs referred to the populations using Ws to realize the accessibility when they participated in all the activities and social affairs in daily life, as well as that WUC was composed of WUs, Ws and NSs. Moreover, according to whether the NSs were needed, this system was divided into two basic forms: WUs-Ws system and WUs-Ws-NSs system. Then the characteristics of WUs, Ws, NSs and the whole system were analyzed and elaborated, and the main characteristics of WUs-Ws system and WUs-Ws-NSs system were clarified. Finally, this paper also put forward two concepts of "instrumental auxiliary ability" and "service auxiliary ability" as well as pointed out that the comprehensive abilities of WUs in WUs-Ws-NSs system was the incorporation of their own abilities, instrumental auxiliary abilities from Ws and the service auxiliary abilities from NSs.

\section{Acknowledgements}

The authors are grateful to the Modern Design and Culture Research Center of the Key Research Base of Philosophical and Social Science in Sichuan, China for supporting this research under grant MD15E027.

\section{References}

[1] State Bureau of Quality and Technical Supervision (SBQTS) (2000) GB/T 14729-2000 Wheelchair-Terms. Standards Press of China, Beijing.

[2] Bellis M. (2014) History of the Wheelchair. http://inventors.about.com/od/wstartinventions/a/wheelchair.htm

[3] Shanghai Hubang Medical Appliances Co., Ltd. (HUBANG) (2012) The Origin of Wheelchair. Disability in China, No. 1,70 .

[4] Rehabilitation Engineering and Assistive Technology Society of North America (RESNA) (2012) RESNA POSITION 
on the application of Ultralight Manual Wheelchairs. RESNA, Arlington.

[5] He, Q.H., Huang, S.P., and Huang, Z.X. (2003) Current Situation and Development Trend of Intelligent Wheelchair. Robot Technique and Application, No. 2, 12-16.

[6] Lu, T., Yuan, K., and Zhu, H.B. (2008) Current Situation and Development Trend of Intelligent Wheelchair. Robot Technique and Application, No. 2, 1-5.

[7] Kauzlarich, J.J. and Thacker, J.G. (1985) Wheelchair Tire Rolling Resistance and Fatigue. Journal of Rehabilitation Research and Development, 22, 25-41.

[8] Kauzlarich, J.J. and Thacker, J.G. (1987) A Theory of Wheelchair Wheelie Performance. Journal of Rehabilitation Research and Development, 24, 67-80.

[9] Brubaker, C.E. (1986) Wheelchair Prescription: An Analysis of Factors That Affect Mobility and Performance. Journal of Rehabilitation Research and Development, 23, 19-26.

[10] Cooper, B.A., Cohen, U., and Hasselkus, B.R. (1991) Barrier-Free design: A Review and Critique of the Occupational Therapy Perspective. American Journal of Occupational Therapy, 45, 344-350.

[11] Oh, S., Hata, N., and Hori, Y. (2005) Control Developments for Wheelchairs in Slope Environments. Proceedings of the American Control Conference, Portland, 8-10 June 2005, 739-744.

[12] Simpson, R.C. (2005) Smart Wheelchairs: A Literature review. Journal of Rehabilitation Research and Development, 42, 423-436.

[13] Xu, Q., Zhu, X.Z., and Zhang, J.G. (2009) Structure Design of Multifunctional Wheel chair and ITS Finite Element Analysis. Mechinery Design and Manufacture, No. 7, 24-26.

[14] Wu, X.L. and Chen, H.J. (2012) Designing Implementation and Engineering Analysis of a Rental Wheelchair. Chinese Journal of Engineering Design, 19, 323-328.

[15] How. T.V., Wang, R.H. and Mihailidis, A. (2013) Evaluation of an Intelligent Wheelchair System for Older Adults with Cognitive Impairments. Journal of Neuro Engineering and Rehabilitation, 10, 1-16.

[16] Garber, S.L. (1985) Wheelchair Cushions: A Historical review. The American Journal of Occupational Therapy, 39, 453-459.

[17] Ferrarin, M., Andreoni, G. and Pedotti, A. (2000) Comparative Biomechanical Evaluation of Different Wheelchair Seat Cushions. Journal of Rehabilitation Research and Development, 37, 315-324.

[18] Rozendaal, L.A., Veeger, H.E.J. and van der Doube, L.H.V. (2003) The Push Force Pattern in Manual Wheelchair Propulsion as a Balance between Cost and Effect. Journal of Biomechanics, 36, 239-247.

[19] Ren, Y., Zhang, J.X., Zhang, J.G., and Hu, J. (2009) Design of Electric Wheelchair for Stance and Supine Posture. Journal of Tianjin University of Science and Technology, 24, 47-49.

[20] Wang, R.H.L. (2011) Enabling Power Wheelchair Mobility with Long-Term Care Home Residents with Cognitive Impairments. Ph.D. Thesis, University of Toronto, Ontario. https://tspace.library.utoronto.ca/bitstream/1807/29906/1/Wang_Rosalie_HL_201106_PhD_thesis.pdf

[21] Perks, B.A., Mackintosh, R., Stewart, C.P. and Bardsley, G.I. (1994) A Survey of Marginal Wheelchair Users. Journal of Rehabilitation Research and Development, 31, 297-302.

[22] Elaine, C.M., Susan, K., Patrick, D.L. and Erin, M. (2002) Physical Functional Performance in Persons Using a Manual Wheelchair. Journal of Orthopaedic and Sports Physical Therapy, 32, 104-113.

[23] Stanley, R.K., Stafford, D.J., Rasch, E. and Rodgers, M.M. (2003) Development of a Functional Assessment Measure for Manual Wheelchair Users. Journal of Rehabilitation Research and Development, 40, 301-307.

[24] Kilkens, O.J.E., Post, M.W., Dallmeijer, A.J., Seelen, H.A. and Lh, V.D.W. (2003) Wheelchair Skills Tests: A Systematic Review. Clinical Rehabilitation, 17, 418-30.

[25] Kilkens O.J.E. (2005) Manual Wheelchair Skill Performance of Persons with Spinal Cord Injuries. Ph.D. Thesis, Maastricht University, Maastricht. http://www.rug.nl/staff/l.h.v.van.der.woude/osnat.pdf

[26] van der Woude, L.H., de Groot, G., Hollander, A.P., van Ingen, S.G.J. and Rozendal, R.H. (1986) Wheelchair Ergonomics and Physiological Testing of Prototypes. Ergonomics, 29, 1561-1573.

[27] Mason, B. (2011) The Ergonomics of Wheelchair Configuration for Optimal Sport Performance. Ph.D. Thesis, Loughborough University, Leicestershire. http://www.rug.nl/staff/l.h.v.van.der.woude/thesisbarry.pdf

[28] Richter, W.M. (2002) The Effect of Seat Position on Manual Wheelchair Propulsion Biomechanics: A Quasi-Static Model-Based Approach. Medical Engineering and Physics, 23, 707-712.

[29] Rodgers, M.M., Tummarakota, S. and Lieh, J. (1998) Three-Dimensional Dynamic Analysis of Wheelchair Propulsion. Journal of Applied Biomechanics, 14, 80-92. 
[30] Kotajarvi, B.R., Sabick, M.B., An, K.N., Zhao, K.D., Kaufman, K.R. and Basford, J.R. (2004) The Effect of Seat Position on Wheelchair Propulsion Biomechanics. Journal of Rehabilitation Research and Development, 41, 403-414.

[31] Finley, M.A., Rasch, E.K., Keyser, R.E. and Rodgers, M.M. (2004) The Biomechanics of Wheelchair Propulsion in Individuals with and without Upper-Limb Impairment. Journal of Rehabilitation Research and Development, 41, 385395.

[32] Yang, Y.S. (2005) Effects of Functional Electrical Stimulation on Trunk Musculature during Wheelchair Propulsion. Ph.D. Thesis, University of Pittsburgh, Pittsburgh. http://d-scholarship.pitt.edu/9552/1/Dissertation Yang 2005.pdf

[33] Dalhousie University (2012) Wheelchair Skills Program (WSP). http://www.wheelchairskillsprogram.ca

[34] Dalhousie University (2013) Wheelchair Skills Program (WSP) Version 4.2: Wheelchair Skills Test Manual. http://www.wheelchairskillsprogram.ca/eng/documents/WST_Manual_version_4.2.1_approved.pdf

[35] Lukersmith, S. (2011) Guidelines for the Prescription of a Seated Wheelchair or Mobility Scooter for People with a Traumatic Brain Injury or Spinal Cord Injury. Lifetime Care and Support Authority, Enable NSW, Sydney.

[36] Fan, W.K. and Hu, Y.S. (2008) Review of Assessment of Activities of Daily Living. Chinese Journal of Physical Medicine and Rehabilitation, 30, 126-129.

[37] General Administration of Quality Supervision, Inspection and Quarantine of the People's Republic of China (GAQSIQ), and Standardization Administration of the People's Republic of China (SAC) (2006) GB 12995-2006 Combustion-Motor-Driven Wheelchairs. Standards Press of China, Beijing. 